\title{
micromachines
}

ISSN 2072-666X

www.mdpi.com/journal/micromachines

Article

\section{Multiplex, Quantitative, Reverse Transcription PCR Detection of Influenza Viruses Using Droplet Microfluidic Technology}

\author{
Ravi Prakash 1, Kanti Pabbaraju ${ }^{2}$, Sallene Wong ${ }^{2}$, Anita Wong ${ }^{2}$, Raymond Tellier ${ }^{2,3}$ and \\ Karan V. I. S. Kaler ${ }^{1, *}$
}

1 Biosystems Research and Applications Group, Department of Electrical and Computer Engineering, Schulich School of Engineering, University of Calgary, Calgary, AB T2N 1N4, Canada;

E-Mail: rprakash@ucalgary.ca

2 Provincial Laboratory for Public Health of Alberta, Calgary, AB T2N 4W4, Canada; E-Mails: kanti.pabbaraju2@albertahealthservices.ca (K.P.); sallene.wong@albertahealthservices.ca (S.W.); anita.wong@albertahealthservices.ca (A.W.); raymond.tellier@albertahealthservices.ca (R.T.)

3 Department of Microbiology, Immunology and Infectious Diseases, Cumming School of Medicine, University of Calgary, Calgary, AB T2N 1N4, Canada

* Author to whom correspondence should be addressed; E-Mail: kaler@ucalgary.ca; Tel.: +1-403-220-5809; Fax: +1-403-220-6855.

Academic Editor: Joost Lötters

Received: 18 November 2014 / Accepted: 16 December 2014 / Published: 23 December 2014

\begin{abstract}
Quantitative, reverse transcription, polymerase chain reaction ( $\mathrm{qRT}-\mathrm{PCR}$ ) is facilitated by leveraging droplet microfluidic (DMF) system, which due to its precision dispensing and sample handling capabilities at microliter and lower volumes has emerged as a popular method for miniaturization of the PCR platform. This work substantially improves and extends the functional capabilities of our previously demonstrated single qRT-PCR micro-chip, which utilized a combination of electrostatic and electrowetting droplet actuation. In the reported work we illustrate a spatially multiplexed micro-device that is capable of conducting up to eight parallel, real-time PCR reactions per usage, with adjustable control on the PCR thermal cycling parameters (both process time and temperature set-points). This micro-device has been utilized to detect and quantify the presence of two clinically relevant respiratory viruses, Influenza A and Influenza B, in human samples (nasopharyngeal swabs, throat swabs). The device performed accurate detection and quantification of the two respiratory viruses, over several orders of RNA copy counts, in unknown (blind) panels of extracted patient samples with acceptably high PCR efficiency (>94\%). The multi-stage qRT-PCR assays on eight panel patient samples
\end{abstract}


were accomplished within 35-40 min, with a detection limit for the target Influenza virus RNAs estimated to be less than 10 RNA copies per reaction.

Keywords: reverse transcription PCR (RT-PCR); Influenza viruses; droplet microfluidics (DMF); nano-texture; dielectrophoresis (DEP); electrostatics; electrowetting (EW); multiplex qRT-PCR assays

\section{Introduction}

The detection of clinically relevant viral pathogens is an essential task performed by medical microbiology laboratories, to help establish diagnosis, guide the subsequent treatment and contribute to public health surveillance, including monitoring of emerging agents. The introduction of molecular detection methods and especially nucleic acid amplification techniques, such as polymerase chain reaction (PCR) [1,2], has revolutionized the capabilities of diagnostic virology laboratories in large part due to the increased sensitivity of detection and improved turnaround time. Since different viruses can have similar clinical presentations, patients typically have to be tested for the presence of several different viruses at the same time; this is leading increasingly to the setting up of testing panels for several viruses linked to a specific syndrome. Some typical examples include gastroenteritis virus panels, viral encephalitis panels and of course respiratory virus panels. In practice, "panels" can be performed in two different ways; several different assays on a single sample with multiple targets/markers by using spectral multiplexing or, by utilizing spatial multiplexing based parallel PCR assays where separate individual sample and target mixtures are prepared and amplified in parallel [3].

PCR is used in the screening and detection of numerous infectious viral or bacterial species, by amplifying the target nucleic acids extracted from patient sample, over several orders of magnitude [1,2]. Quantitative polymerase chain reaction (qPCR) furthermore facilitates real-time detection of target nucleic acid during the amplification process and allows for quantitation of the initial amount of template $[4,5]$. PCR at microscale leads to a reduction in the reaction time, bio-sample/reagent volume [6,7]. Following the early development of the conventional close channel microfluidics based PCR micro-devices [6-9], handling the PCR sample volume in form of rapidly dispensed, discrete microliter or smaller droplets has become a preferred method of choice for PCR micro-devices [10,11]. Most microfluidic PCR systems have focused primarily on either reducing the PCR reaction volume (down to few hundred nanoliter) [12-14] or reducing the PCR reaction time ( 10 $\mathrm{min})$ [12-16]. The requirement of high surfactant concentration in the continuous oil phase to stabilize the PCR droplets, lack of individual addressing of the multiple droplets and the excessive need for off-chip overhead (pumps, plumbing, valves) are just a few of the challenging issues driving the development of miniaturized PCR set-ups towards droplet microfluidic (DMF). Among the available DMF methods, electro-actuation of droplets on patterned substrates (Glass, Silicon, Polymer, etc.) is the most effective means of precision dispensing and subsequently handling multitude of bio-sample and reagents using a miniaturized device. Electrowetting (EW) or, EW-on-dielectric (EWOD) [17,18] has been successfully utilized to demonstrate PCR reactions at microscale [19-22], however, the necessity of active electrode switching to facilitate droplet motion, in the digital microfluidic technique, results in a substantial 
electrical overhead, especially for the multiplexed, chip based bio-assay schemes. Recently, our work has demonstrated that a continuous droplet transport scheme, which enables droplet transport and thermal cycling without the requirement of active electrode switching [11], can be an effective solution for a PCR micro-device with reduced electrical overhead requirement for a multiplexed diagnosis system. Here active droplet transport is facilitated by electrostatic or, droplet-dielectrophoresis (D-DEP) based electro-actuation technique, which utilizes herring-bone shaped electrode arrays to facilitate droplet transport and thermal cycling [11,23,24].

In cases pertaining to the detection and quantification of RNA viruses, for example Influenza viruses, Hepatitis $\mathrm{C}$ virus, Measles virus, SARS-CoV and Ebola virus, detection by PCR requires transcribing the viral RNA extracted from virions through a reverse transcription reaction, to yield complementary DNA (cDNA) molecules. Apart from virology, other major applications of RT-PCR include analysis of gene expression from target cells and detection of certain genetic diseases. In quantitative analysis of RT-PCR (qRT-PCR), a reaction mixture containing both a reverse transcriptase enzyme and a thermo-stable DNA polymerase (TAQ) is used so that the two enzymatic reactions (reverse transcription and PCR amplification) can be performed serially through temperature control, as an integrated two-step process. On a miniaturized scale, RT-PCR reactions have been achieved by utilizing microfluidic methods for manipulating nucleic acid samples and PCR reagents including the use of continuous flow techniques [6-9] and discrete droplet based microfluidics [10,11]. The majority of microfluidic implementations for RT-PCR assays have been targeted to gene expression analysis, where a large amount of genomic molecules has helped towards lowering the reaction volumes to sub-microliters [12-14]. However, in clinical diagnostic applications, for the detection of trace quantities of viral RNA in a matrix sample that often contains an abundance of genomic DNA from the human host, the emphasis is placed on the reliable, rapid detection [15].

The evolution of PCR technologies over the last two decades suggests the need for further improvement towards the performance and reliability of PCR systems. The droplet digital PCR (ddPCR ${ }^{\mathrm{TM}}$, Bio-Rad, Hercules, CA, USA) system commercially available from Bio-Rad incorporates the close-channel microfluidic based droplet generation method to create a large library $(\sim 15,000)$ of sub-microliter droplets, which are dispensed from a large PCR sample/reagent mixture $(\sim 20 \mu \mathrm{L})[16]$. This approach allows for one step detection and quantification of extracted nucleic acid and it is an excellent example of PCR technology that illustrates the feasible integration of microfluidics into such commercial systems.

Reports of large PCR sample arrays of sub-microliter reactions have also been reported for high-throughput qRT-PCR, with as few as five copies of template RNA in each reaction [14]. However, detection of a panel of infectious viruses (such as the respiratory virus panel) in human samples utilizing a qRT-PCR microchip remains to be realized. Examples of recent single RT-PCR applications using microfluidics suggest a detection time (reverse transcription and thermal cycling) of up to an hour, using as low as $2-5 \mu \mathrm{L}$ PCR volume $[14,15]$. We have previously designed a microchip utilizing electrostatic/droplet-DEP (D-DEP) electro-actuation method and integrated thermostatic zones (micro-heaters and resistive temperature sensors) to achieve single qRT-PCR amplification of in vitro synthesized Influenza viral RNA [11], with a detection threshold of less than 10 copies of template RNA in the PCR reaction volume. We have also investigated the scalability of PCR sample volume in our device application, over the range of $1-10 \mu \mathrm{L}$ [11], which is industrially accepted for viral detection in clinical samples. In this work, we have modified the previously designed continuous, 
D-DEP electrode architecture for the PCR thermal cycling to produce a spatially multiplexed PCR micro-device, suitable for carrying out several different qRT-PCR reactions in parallel (up to eight assays per chip) and with a built-in flexibility to accommodate different cycling parameters for each reaction. The performance of this micro-device is illustrated by the parallel execution of assays for the detection of Influenza A virus and Influenza B virus in different panels of clinical samples. The reported multiplexed qRT-PCR assays are a first demonstration of a D-DEP based DMF device for analysing multiple clinically relevant viral pathogens in panels of extracted patient samples.

\section{Experimental Section}

\subsection{Device Fabrication}

The micro-device was designed using the MEMSPro L-Edit (v. 8.0) CAD software. The DMF chip was fabricated at a micro/nano fabrication facility (Nanofab, Edmonton, Canada). The detailed fabrication method has been previously reported $[11,25]$. The fabrication procedure utilized to produce the micro-device are identical to the one used in the development of single qRT-PCR microchips, shown in Figure 1a [11]. A pair of $6.6 \mathrm{~cm} \times 3.0 \mathrm{~cm}$ micro-devices was fabricated from a $10 \mathrm{~cm}$ square glass (Borofloat) wafer (Figure 1b,c). The micro-device consists of: (1) an array of photo lithographically patterned chromium (Cr thickness: $200 \mathrm{~nm}$ ) micro-heaters and resistance temperature detectors (RTDs) to create the two thermostatic zones (Heater blocks 1 and 2) required during the thermal cycling, (2) a photo lithographically patterned gold/chrome overlay (100 nm Au/ $200 \mathrm{~nm} \mathrm{Cr}$ ) for electrical connections to the micro-heaters/RTD sensors, (3) another photo lithographically patterned Aluminum (200 nm) layer for D-DEP electrodes and (4) $\mathrm{Au} / \mathrm{Cr}$ metallization for the EW track, utilized for loading the PCR template and reagent mix droplets to the thermal cycler electrodes. These 3 different metal layers were electrically isolated and passivated using dielectric stacks of silicon nitride ( $\mathrm{Si}_{3} \mathrm{~N}_{4}$ thickness: $500 \mathrm{~nm}$ ), to prevent sample electrolysis during electro-actuations. The very top dielectric layer was furthermore utilized to produce a nano-textured super hydrophobic $(\mathrm{SH})$ top surface, utilizing a soft lithography technique [25]. The SH surface provided a high droplet contact angle $\left(\mathrm{CA} \sim 156^{\circ}\right)$ during the device application and significantly minimizes bio-sample adsorption $[11,25]$.

Figure 1a demonstrates the first generation single qRT-PCR microchip, which facilitates spiral droplet transport between the two heater blocks [11]. This electrode structure required up to $10 \mathrm{~s}$ to convey the droplet from one thermal zone to another. This delay is principally due to the two relay-controlled track switching required to facilitate the spiral droplet transport. While attempting to improve the electrode architecture towards a more compact single cell design which can result in a larger assay matrix from a $10 \mathrm{~cm}$ substrate, it was observed that a single bi-direction track (see Figure 1b significantly reduces the PCR cell area by up to $25 \%$ and facilitates droplet transport from one zone to another in $\sim 5 \mathrm{~s}$ with one track switching (on the end). This coupled with the 25-30 s annealing period (in the lower temperature zone) ensured the reduction of droplet track size and hence the thermal cycling time. Similar to our previous work, a standard fluorescent thermometry dye (Rhodamine B dye) was used to verify the temperature of the droplet during the annealing and denaturation phase of the PCR thermal cycle [26,27]. Figure 1b shows the improved bi-direction electrode structure as part of the multiplexed (eight-plex) qRT-PCR unit (Figure 1c), which was fabricated and utilized in all the experimental work, reported in this paper. 


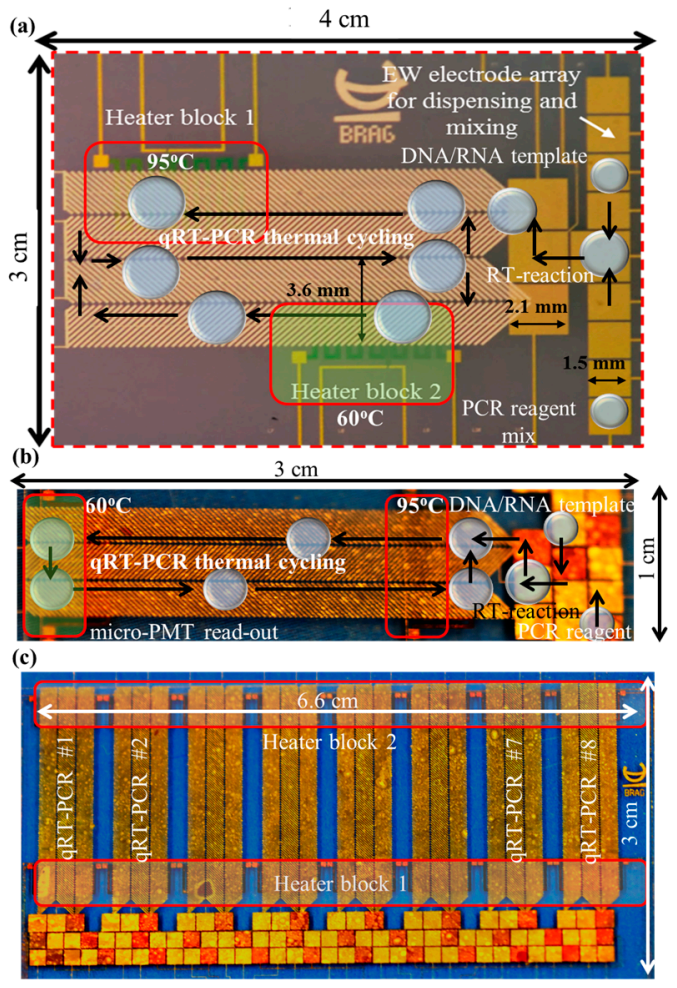

Figure 1. (a) Photomicrographs of (a) the spiral droplet-dielectrophoresis (D-DEP) electrode architecture used in the earlier single quantitative, reverse transcription, polymerase chain reaction (qRT-PCR micro-device) [11]; (b) the continuous, bi-directional droplet actuation scheme and; (c) the eight-plex micro-device.

\subsection{Sample Preparation}

The various sample preparation protocols used in this work are detailed below.

a. Extraction of total nucleic acid from clinical specimens. The extracted nucleic acids, including RNA, were from left-over samples from patients, initially submitted to ProvLab for Influenza virus detection; nucleic acid extracts from samples were labeled at ProvLab as positive for Influenza A or Influenza B or negative, but were otherwise anonymized. Initially, respiratory samples including nasopharyngeal swabs (NP) and throat swabs (TS) were pre-treated with $25 \mu \mathrm{L}$ of $0.01 \mathrm{mAU} / \mu \mathrm{L}$ of protease (Qiagen, Mississauga, Ontario, Canada) in a thermomixer (Eppendorf, Westbury, NY, USA) at $56{ }^{\circ} \mathrm{C}$ and $1000 \mathrm{rpm}$ for $10 \mathrm{~min}$ and the supernatant was collected for the extraction process. The total nucleic acid was extracted from the treated samples using the easyMAG ${ }^{\circledR}$ automated extractor (bioMérieux, Montreal, Canada) according to the manufacturer's instructions [11]. The extracted nucleic acid was eluted into a final volume of $110 \mu \mathrm{L}$ of elution buffer (Borate buffer; $\mathrm{pH}$ 8.5) from a sample input volume of $200 \mu \mathrm{L}$.

b. qRT-PCR assay. All samples used for validation studies underwent extraction and were tested for Influenza A and Influenza B using real-time RT-PCR assays. The primer and probe sequences from previously reported real-time RT-PCR assays (developed at the Center for Disease Control (CDC), USA) were used for the detection of Influenza A and Influenza B viral RNA. The Influenza A assay targets the matrix gene and the Influenza B assay targets the non-structural gene resulting in the amplification of a 105 base pair product for influenza A and 103 base pair product for Influenza B. 
Amplification was performed by one-step RT-PCR using the TaqMan ${ }^{\circledR}$ Fast Virus One-Step RT-PCR Master Mix (Life Technologies Inc., Burlington, Canada), $0.8 \mu \mathrm{M}$ each of sense and antisense primers and $0.2 \mu \mathrm{M}$ of the labeled probe. Five microliters of in vitro RNA was combined with $5 \mu \mathrm{L}$ of the master mix. The reaction parameters included a reverse transcription (RT) step performed at $50{ }^{\circ} \mathrm{C}$ for 5 min, followed by enzyme activation at $95{ }^{\circ} \mathrm{C}$ for $20 \mathrm{~s}$. The PCR assay included 45 cycles of denaturation at $95^{\circ} \mathrm{C}$ for $3 \mathrm{~s}$ and annealing/ extension at $60^{\circ} \mathrm{C}$ for $20 \mathrm{~s}$.

c. In-vitro RNA and blind panel samples. To synthesize in vitro RNA of Influenza A and Influenza $B$ viruses, primers flanking the detection region were used to amplify fragments of the $M$ gene including the region targeted by the primers and probes in the real-time PCR assays. The PCR products were cloned using the TOPO TACloning Dual Promoter Kit (Life Technologies, Burlington, Canada) and the plasmid DNA linearized using restriction enzymes (Hind III) and transcribed using the T7 RiboMAXTM Express (Promega, Madison, WI, USA). The resultant in vitro transcribed RNA was quantified and serial dilutions were utilized for the standard quantification process.

Validation studies were performed using a total of three blind panels: 1, A panel of six NP samples that had previously tested either positive or negative for Influenza A with a range of viral loads (crossing threshold $(\mathrm{Ct})$ values ranging from 23 to 33 by qRT-PCR) (Table 1a); 2, A panel of six Influenza A positive NP and TS samples, with a range of viral loads (Crossing threshold values ranging from 24 to 32 by qRT-PCR) (Table 1b); and 3, A mixed panel of Influenza A and B positive NP specimens including a co-infected specimen (Table 1c).

Table 1. Tabular list of the three different clinical panels used to validate the performance of multiplexed assays using the fabricated micro-device.

\begin{tabular}{|c|c|c|}
\hline Panel Sample No. & Sample Style & Target \\
\hline \multicolumn{3}{|c|}{ (a) The Influenza A panel samples (End-point PCR) } \\
\hline 1 & Nasopharyngeal Swab & FluA; pdm09 \\
\hline 2 & Nasopharyngeal Swab & Respiratory negative \\
\hline 3 & Nasopharyngeal Swab & Respiratory negative \\
\hline 4 & Nasopharyngeal Swab & FluA; pdm09 \\
\hline 5 & Nasopharyngeal Swab & Respiratory negative \\
\hline 6 & Nasopharyngeal Swab & FluA; pdm09 \\
\hline \multicolumn{3}{|c|}{ (b) The Influenza A blind panel } \\
\hline 1 & Nasopharyngeal Swab & FluA; pdm09 \\
\hline 2 & Nasopharyngeal Swab & FluA; pdm09 \\
\hline 3 & Throat Swab & FluA; pdm09 \\
\hline 4 & Nasopharyngeal Swab & FluA; pdm09 \\
\hline 5 & Nasopharyngeal Swab & FluA; pdm09 \\
\hline 6 & Nasopharyngeal Swab & FluA; pdm09 \\
\hline 7 (+ve control) & H3 M-gene In-vitro RNA & FluA; H3 \\
\hline 8 (-ve control) & PCR water & - \\
\hline \multicolumn{3}{|c|}{ (c) The Influenza A, Influenza B mixed blind panel } \\
\hline 1 & Nasopharyngeal Swab & FluA, FluB \\
\hline 2 & Nasopharyngeal Swab & FluA, FluB \\
\hline 3 & Nasopharyngeal Swab & FluA, FluB \\
\hline 4 & Nasopharyngeal Swab & FluA, FluB \\
\hline
\end{tabular}




\subsection{Experimental Procedures}

A schematic diagram of the experimental set-up is shown in Figure 2a. The set-up consists of the required optical components, a microchip-PCB (Printed Circuit Board) assembly secured on a motorized $x y z$ stage, an (field programmable gate array) FPGA interfaced NI PXIe-1062Q (National Instruments, Austin, TX, USA) unit for electro-actuation and feedback control, a micro-photomultiplier tube ( $\mu$ PMT, Hamamatsu, Japan) for continuous, scanning mode, real-time fluorescence signal read-out of the panel assays. Although it is not a packaged unit, the set-up already shows miniaturization of the multiplexed PCR unit, which is driven by the NI PXIe unit (National Instrument, Austin, TX, USA). The optical components are currently housed on a microscope platform and include: microPMT (H12400-00-01) for parallel read-out; a color charge-coupled device (CCD) camera (QImaging, Surrey, Canada) and a high speed complementary metal oxide semiconductor (CMOS) camera (Canadian Photonics Lab, Manitoba, Canada) for visual inspection and video/image capturing; a motorized $x y z$ stage, controlled by an OptiScan unit (Prior Scientific) via NI program for rapid scanning and panel PCR read-outs. The operation of the resistive thermostatic zones through the NI PXIe unit has been previously described [11]. The microchip-PCB assembly (Figure 2b) utilizes a PCB (manufactured at AP Circuits, Calgary, Canada) mounted PCI ZIF test connector (Meritec Inc., Painesville, OH, USA) to secure and address the various electro-actuations and feedback controls during the multiplexed assays.

Various photomicrographs of the droplet electro-actuation based PCR thermal cycling, over the micro-device shown in Figure 1c, are illustrated in Figure 3. For all the qRT-PCR assays reported in this work, we have utilized a sealed enclosure containing PCR grade mineral oil (bioMerieux, Montreal, Canada), secured within a heated indium tin oxide (ITO)/Glass top plate, the bottom substrate and a plexiglass fixture.

The substrate was maintained at a temperature of $50{ }^{\circ} \mathrm{C}$, required for the $\mathrm{RT}$ reaction which takes place on the EW electrode array, following the mixing of PCR sample and reagent droplets (see Figure 1). Furthermore to minimize thermal diffusion from the PCR droplets, the ITO/Glass top plate was also maintained at the same temperature using an isothermal plate, as shown in Figure 2b [11]. The PCR reaction volume for all the qRT-PCR assays reported in this work was kept constant at $10 \mu \mathrm{L}$, in order to facilitate validation studies using commercial qRT-PCR equipment at ProvLab. For each multiplexed assay, extracted RNA sample droplet $(5 \mu \mathrm{L})$ and PCR reagent mixture droplets $(5 \mu \mathrm{L})$ were manually pipetted and mixed using the EW electrode array, as shown in Figure 1a,b. Figure 3a shows the continuous, bi-directional actuation of a $10 \mu \mathrm{L}$ PCR droplet following the EW based dispensing and mixing. The electrostatic/D-DEP actuation $[11,23,24]$ is facilitated by an AC voltage (50-60 Vpp, $40 \mathrm{~Hz}$ ), applied across a pair of herringbone electrodes upon which the droplet is electrically confined and transported. The droplet track is switched with a $50 \mathrm{~V} \mathrm{DC}$ voltage applied across the top and bottom herringbone electrode pair to facilitate droplet transfer between the two temperature zones. Although the track switching is manually achieved in a timed fashion (DC bias applied after $6 \mathrm{~s}$ on either end of linear D-DEP actuation), it is fairly reliable due to the short track lengths and controlled droplet speed [11]. Figure 3b further illustrates the parallel thermal cycling of two identical sized $(10 \mu \mathrm{L})$ PCR droplets, following the reverse transcription step, during a multiplexed qRT-PCR assay. The apparent increase of droplet size during the denaturing phase is 
expected due to the increased thermal stress that the droplet is subjected as it heats up to the higher temperature set-point. As the droplet moves out of the denaturing zone, it retains its original high contact angle, hence enabling reliable transport during multiple thermal cycles (see Figure $3 \mathrm{~b}$ ). The transport of droplets between the two thermostatic temperature zones is achieved in $\sim 5-6 \mathrm{~s}$, resulting in an effective temperature ramp rate of $\sim 5^{\circ} \mathrm{C} / \mathrm{s}$. The PMT read-out is carried out over the annealing zone (at $60{ }^{\circ} \mathrm{C}$ ), using a linear scan of the multiple droplets, with an optical aperture set higher than the droplet diameter (twice as large as the droplet diameter) to ensure complete capture of the fluorescent signal from each droplet during the linear scan. The entire linear scan requires up to $25 \mathrm{~s}$ for the complete array of eight assay droplets. The captured fluorescent signal is adjusted with the background photocurrent value and plotted vs. PCR cycle number to obtain the complete PCR curve, reported in the results section.

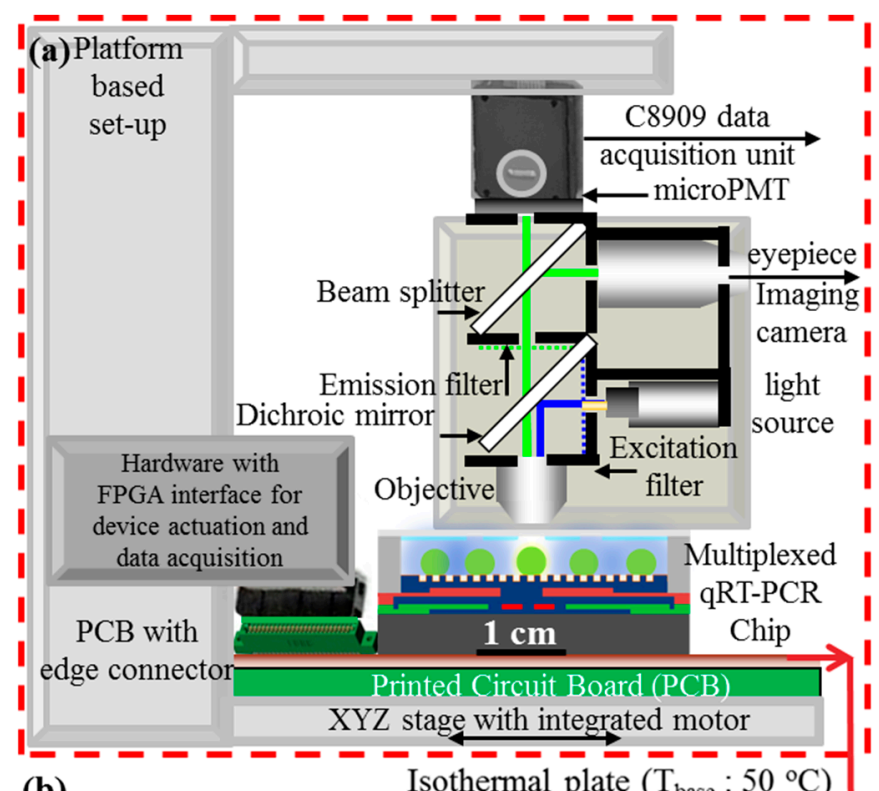

(b)

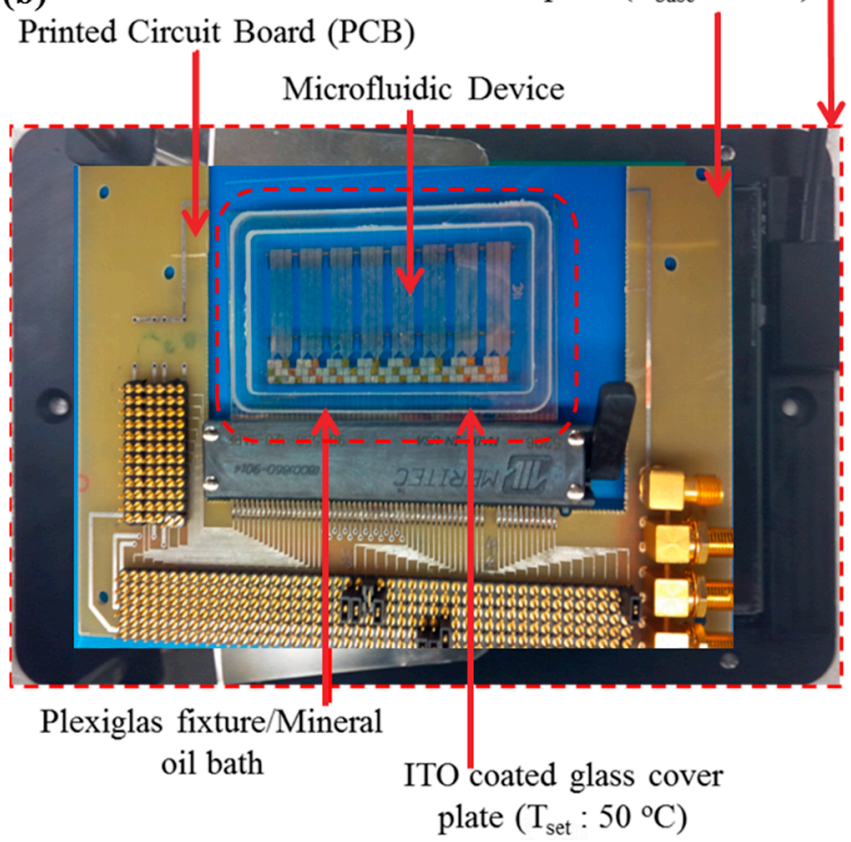

Figure 2. (a) Schematic diagram of the experimental setup; (b) An image of the microchip-PCB (Printed Circuit Board) fixture. 
(a) Bi-directional, continuous droplet transport on D-DEP electrode structure

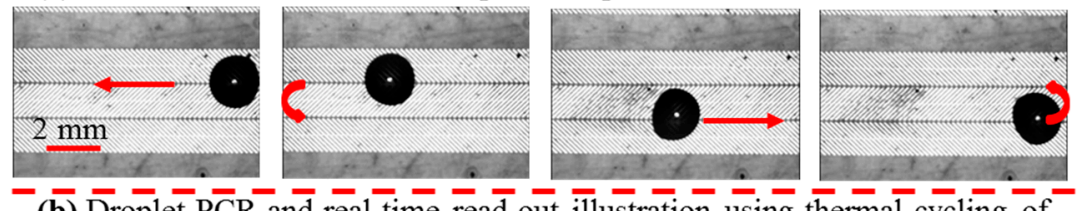

(b) Droplet $\bar{P} \bar{C} R$ and real-time read-out illustration using thermal cycling of two $10 \mu \mathrm{L}$ qRT-PCR sample droplets

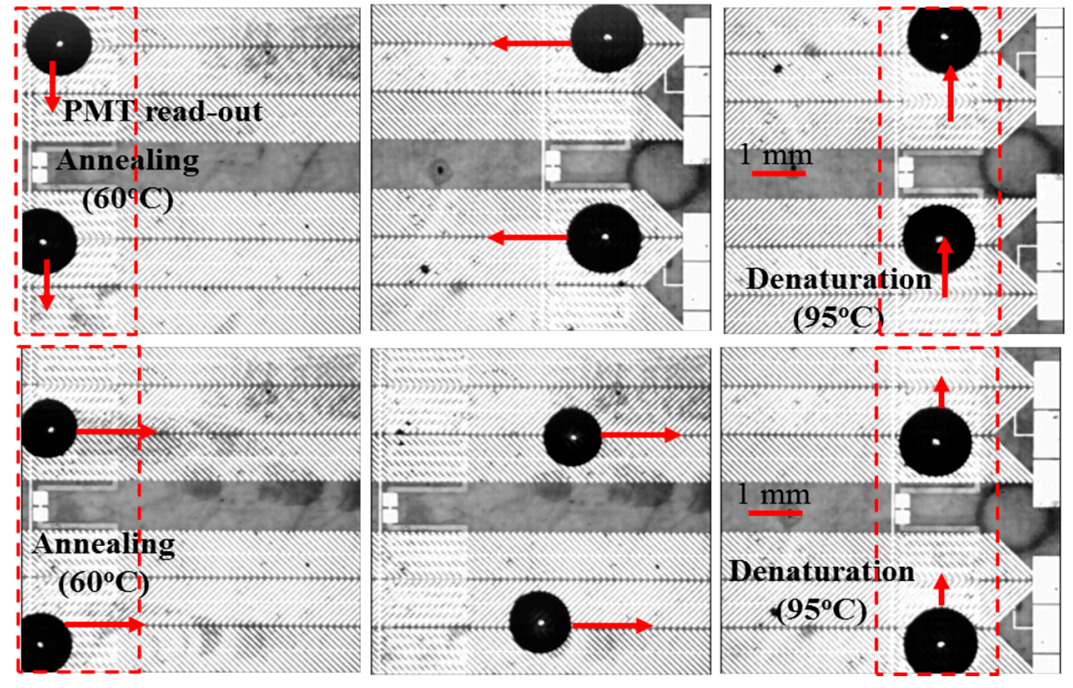

Figure 3. Photomicrographs of (a) the different phases of continuous droplet transport over the newly designed bi-directional electrode scheme and (b) frames extracted from a real-time video showing different stages during qRT-PCR thermal cycling using two $10 \mu \mathrm{L}$ polymerase chain reaction (PCR) droplets on a segment of the micro-device.

\section{Results and Discussion}

In order to validate the operation and performance of the micro-device, both end-point and quantitative RT-PCR assays were carried out on three different panels of clinically extracted patient samples (see Table 1).

\subsection{Standard Quantification Curves for qRT-PCR Amplification of Spiked Influenza A and Influenza B RNA Samples}

A key feature of qRT-PCR equipment is its ability to perform quantitative PCR amplification of target nucleic acid in matrix samples, with a high degree of accuracy and repeatability, over several orders of magnitude of initial template concentration. This allows the user to reliably infer the initial target DNA/RNA concentration from the qRT-PCR plots. In order to test the performance of our micro-device and furthermore to deliver quantitative outcomes on clinical samples, we used spiked in vitro RNA solutions, which were serially diluted and amplified simultaneously on the multiplexed array. The stock in vitro RNA solutions for Influenza A and B viruses were prepared as detailed in Section 2.2. The attributes of the resultant spiked samples are reported in Figure 4, which also presents the extracted qRT-PCR curves obtained for each of the 10 spiked samples (five Influenza A and five Influenza B RNA samples, shown in Figure 4). For analyzing the threshold cycle (Ct) value throughout this paper, we have set the threshold signal level based on the fluorescent noise floor of the negative control sample (see Figure 4a,b). The Ct values (averaged over two sets of multiplexed assays) were 
then plotted versus the natural log of the RNA concentration (Copy count), to report the standard quantification curves for the two target viruses (Figure 4c). The error bars, shown in the plots reported in Figure 4 and all following PCR curves, were calculated as standard deviation data from two different sets of qRT-PCR assays, conducted over two different micro-devices.

The slope $(m)$ of the linear curve in Figure $4 c$ is related to the efficiency $(E)$ of the PCR as [28]:

$$
E=10^{-1 / m}-1
$$

Based on Equation (1), the PCR efficiency for the Influenza A RNA samples was found to be $\sim 95.4 \%$ whereas, the PCR efficiency for the Influenza B RNA samples was $\sim 94.6 \%$. The outcomes of these experiments confirmed that the designed micro-device can reliably achieve parallel and high efficiency qRT-PCR assays on multiple nucleic acid samples. Having confirmed the PCR efficiency of the micro-device, it was then used to detect the viral RNA from extracted nucleic acids from clinical samples at the ProvLab Calgary (see Section 2.2).
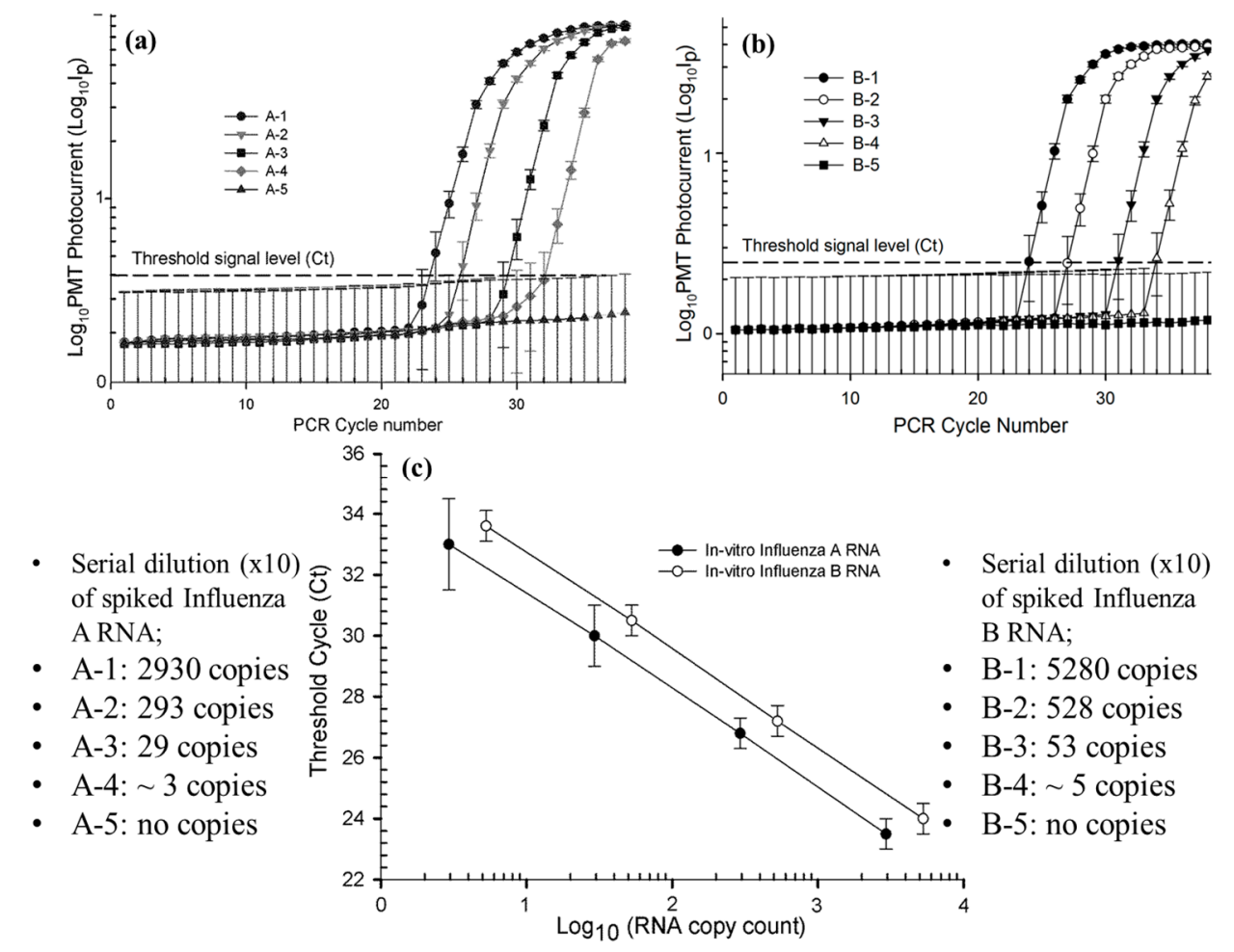

Figure 4. qRT-PCR amplification plots of (a) spiked Influenza A samples, (b) spiked Influenza B samples and (c) standard quantification curves for spiked Influenza A and B. samples (photocurrent, $I_{p}$ in $\mu \mathrm{A}$ ).

\subsection{End-Point, RT-PCR Assay for a Clinical Panel of Influenza A RNA Virus}

The first of the three panel assays conducted during this work used extracts from clinical samples previously characterized reported in Table 1a. A $5 \mu \mathrm{L}$ droplet of extract from each of the six samples, along with an in vitro RNA sample (positive control) and a RNA free water sample (negative control) were sequentially loaded onto the respective sites (see Figure $2 b$ ) and mixed with $5 \mu \mathrm{L}$ of PCR reagent droplets. The combined $10 \mu \mathrm{L}$ PCR droplet was maintained at $50{ }^{\circ} \mathrm{C}$ for $5 \mathrm{~min}$, for completion of the RT-reaction, before initiating parallel PCR assays. 
Once the RT-reaction was complete, the eight samples were simultaneously thermally cycled for 38 PCR cycles. The motorized stage was only used at three set-points (after cycle \# 10, 25 and 38) to extract the PMT photo-current read-out (see Table 2) and the PCR end-points were also recorded as CCD images, shown in Figure 5. The outcomes of this end-point parallel PCR assays, as illustrated in Figure 5 and Table 2 indicate successful identification of the eight panel samples, with the fluorescence readings and $\mathrm{CCD}$ images identifying samples 1, 4, 6 and 7 (+ve control) that tested positive for Influenza A virus. The three set-point PMT readings to some extent relate to the initial RNA concentration of the different panel samples as seen from Table 2.

The aberrations evident in this and other following CCD fluorescent images of PCR droplets is a result of diffraction of incident light onto locally coagulated nano-beads, which is a by-product of the soft-lithography based nano-texturing process, used during the device fabrication [25]. However, the effect of such aberrations are measured and accommodated for as the background signal levels in the PCR curves, which remain fairly constant as evident in the PCR curves reported in the following sections.

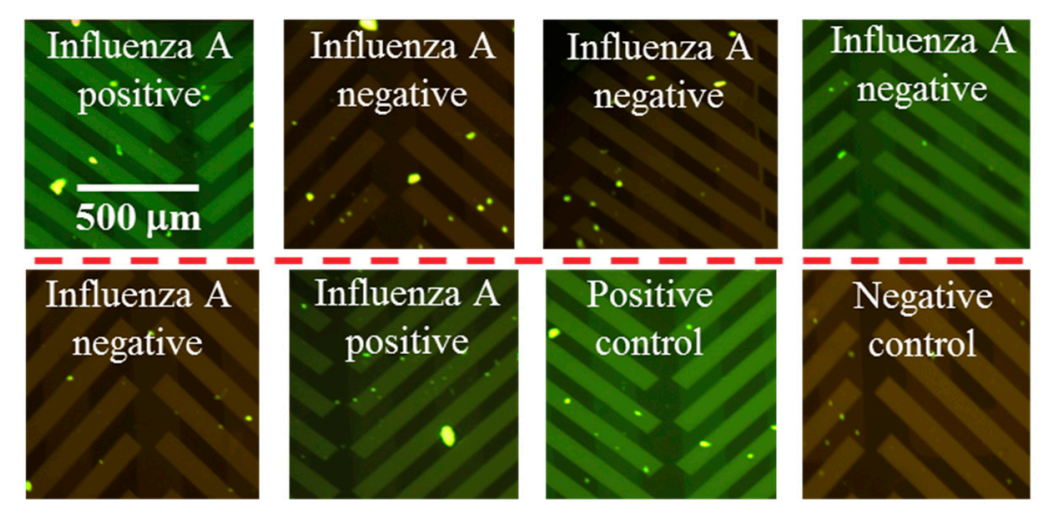

Figure 5. Charge-coupled device (CCD) images showing the outcomes (fluorescent intensity) of the end-point PCR assay carried out using panel samples of Table 1a.

Table 2. Outcomes of the end-point panel polymerase chain reaction (PCR) using samples from Table 1a.

\begin{tabular}{ccccc}
\hline \multirow{2}{*}{ Panel Sample No. } & \multicolumn{3}{c}{ PMT Photocurrent at Different PCR End Points $\left(\boldsymbol{I}_{\mathbf{p}}\right.$ in $\left.\boldsymbol{\mu A}\right)$} & \multirow{2}{*}{ ProvLab $\boldsymbol{C t}$} \\
\cline { 2 - 4 } & PCR cycle \# 10 & PCR cycle \# 25 & PCR cycle \# 38 & \\
\hline 1 & 1.09 & 12.90 & 25.77 & 24 \\
2 & 1.05 & 1.97 & 3.41 & Negative \\
3 & 1.04 & 1.77 & 2.92 & Negative \\
4 & 1.08 & 7.75 & 23.35 & 30 \\
5 & 1.06 & 1.97 & 3.95 & Negative \\
6 & 1.04 & 4.51 & 18.23 & 33 \\
7 (+ve control) & 1.09 & 15.82 & 30.35 & 29 \\
$8(-$ ve control) & 1.06 & 1.85 & 3.01 & Negative \\
\hline
\end{tabular}

\subsection{Quantitative, Multiplexed RT-PCR Assay on an Influenza A blind Panel}

Following the successful analysis of a known panel of extracts from clinical samples using the multiplexed, end-point RT-PCR assay, we then analyzed a panel of clinical samples submitted blindly (described in Table 1b). The blind panel, prepared at ProvLab Calgary, included extracts from patients 
diagnosed with Influenza viral infection. The panel varied in terms of the presence/absence of the RNA virus as well as the concentration of viral load, amongst the eight samples. A positive control (sample \#7) and negative control (RNA free water; sample \#8) were also included in the panel. This panel was subjected to two multiplexed qRT-PCR analyses on two different micro-devices. In both analyses, the motorized stage and PMT modules were used to establish PCR curves from each of the panel samples, which are reported in Figure 6. Following the assay, the chip based PCR curves were plotted and the corresponding $C t$ values for each of the panel samples were analyzed and reported as the average $C t$ over the two micro-device based PCR assays. Subsequently, qRT-PCR reactions on the same panel of samples were also carried out at ProvLab, using the ABI 7500 Fast (Life Technologies Inc., Burlington, Canada) equipment and the $C t$ values from both analyses are compared in Table 3.

As is clear from Table 3 and Figure 6, the outcomes of the parallel, qRT-PCR assay using the eight panel samples on the micro-device are in agreement with the commercial PCR set-up, with accurate identification of each panel samples.

The $C t$ values obtained from the micro-device are in agreement with the $C t$ values yielded by the commercial equipment. It was noticed that the $C t$ values for the micro-device were consistently lower than those obtained at the ProvLab, however the variation and scalability of the two $C t$ value sets are almost identical. The lower $C t$ values for the micro-device can be attributed to a more sensitive detector (PMT compared to a CCD imager used in the commercial set-up). In Table 3, we have also reported the initial RNA copy count in each of the positively identified panel samples, estimated using the standard quantification curve for Influenza A virus RNA, reported in Section 3.1, Figure 4.
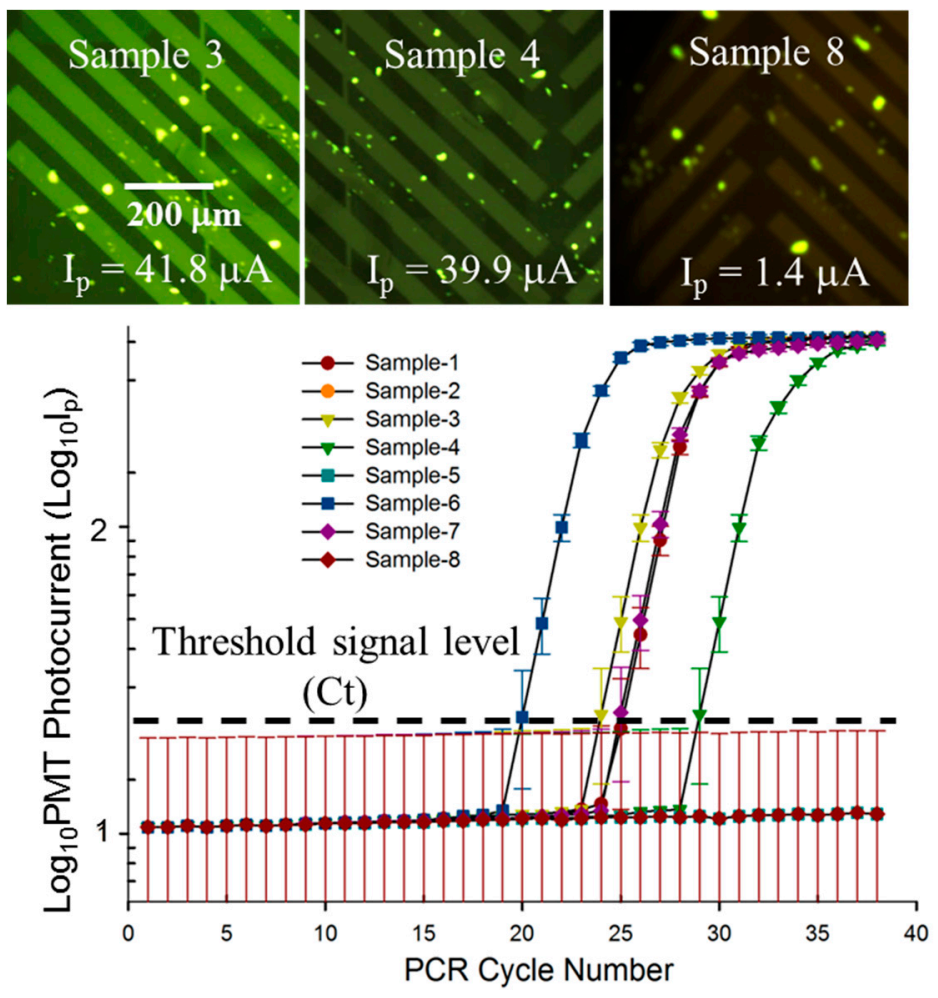

Figure 6. Plot showing qRT-PCR curves obtained during the multiplexed assay using blind panel samples of Table 1b. The fluorescent photomicrographs show a 10× magnified image, centered within the PCR droplets following 38 amplification cycles $\left(I_{\mathrm{p}}\right.$ in $\left.\mu \mathrm{A}\right)$. 
Table 3. Outcomes of the micro-device quantitative, reverse transcription, polymerase chain reaction (qRT-PCR micro-device) assay using panel samples of Table $1 \mathrm{~b}$.

\begin{tabular}{ccccc}
\hline Panel Sample No. & Target & ProvLab $\boldsymbol{C} \boldsymbol{t}$ & Chip $\boldsymbol{C} \boldsymbol{t}$ & Initial Copies of Template RNA \\
\hline 1 & Flu A & 29 & 25 & $\sim 590$ \\
2 & Flu A & Negative & Negative & Not applicable \\
3 & Flu A & 30 & 26 & $\sim 300$ \\
4 & Flu A & 32 & 30 & $\sim 20$ \\
5 & Flu A & Negative & Negative & Not applicable \\
6 & Flu A & 24 & 21 & $\sim 3500$ \\
7 (+ve control) & Flu A & 29 & 26 & $\sim 250$ \\
$8(-$ ve control) & Flu A & Negative & Negative & $\sim 110$ \\
\hline
\end{tabular}

\subsection{Quantitative, Multiplexed RT-PCR Assays on a Mixed, Four Sample Influenza A, Influenza B Blind Panel}

The usual approach to a spectral multiplexed PCR analysis relies on the use of a multitude of primers and probes targeting each of the intended agents to be detected in the same PCR droplet. As a result of the spectral signal bandwidth and optical filtration limitations, this results in practice in limiting the multiplexing capabilities to up to five to six targets per PCR assay. The development of our micro-device was inspired by the notion of incorporating both spectral and spatial multiplexing, where multiple targets can be amplified and read-out in a parallel and automated fashion.

In order to demonstrate this versatile multiple sample target handling, we investigated a mixed blind panel of clinical samples, as shown in Table 1c, which contained different initial concentrations of Influenza A and Influenza B viral RNA, prepared from patient samples extracted at ProvLab Calgary. The synthesized molecular probes for the two RNA targets were labeled respectively with FAM ${ }^{\mathrm{TM}}$ ( $\left.\lambda_{\text {ex. }} / \lambda_{\text {em.: }} 492 \mathrm{~nm} / 520 \mathrm{~nm}\right)$ and VIC ${ }^{\text {TM }}\left(\lambda_{\text {ex. }} / \lambda_{\text {em.: }} 538 \mathrm{~nm} / 554 \mathrm{~nm}\right)$ fluorophores. The four panel samples from Table 1c were then paired in binary combination with the reagent mix droplets containing one of the two fluorescent markers and transported to the eight droplet tracks.

The eight $10 \mu \mathrm{L}$ PCR droplets were then amplified over 38 PCR cycles and analyzed during the annealing phase of each cycle, through the continuous mode PMT read-out. The multiplexed assays (38 PCR cycles and RT reaction), which were repeated on two different micro-devices, were completed within $40 \mathrm{~min}$ from sample/reagent loading onto the micro-device to the determination of all qRT-PCR curves (and the corresponding $C t$ values). The extracted data was plotted and the resulting qRT-PCR curves are reported in Figure 7. After completion of the thermal cycling, fluorescent CCD images were captured showing the eight PCR droplets (see Figure 7). It is clear from the CCD fluorescent images, and from the curves, that sample 1 tested positive for Influenza A virus, sample 2 tested positive for both Influenza A and Influenza B viruses, sample 3 tested negative for both RNA viruses and sample 4 tested positive for Influenza B virus. The $C t$ values, analyzed from the PMT data and averaged over two different micro-device based qRT-PCR assays, are reported in Table 4, alongside the $C t$ values measured with the ABI 7500 fast, at ProvLab Calgary and an estimated initial RNA template copy number. Clearly the multiplexed assay on the micro-device successfully analyzes the mixed blind panel of Influenza A and B viruses and accurately reflected their relative concentrations. 
These findings support our contention that a combination of spatial and spectral multiplexing will significantly extend the current limitations of the conventional multiplexed qRT-PCR methodology.

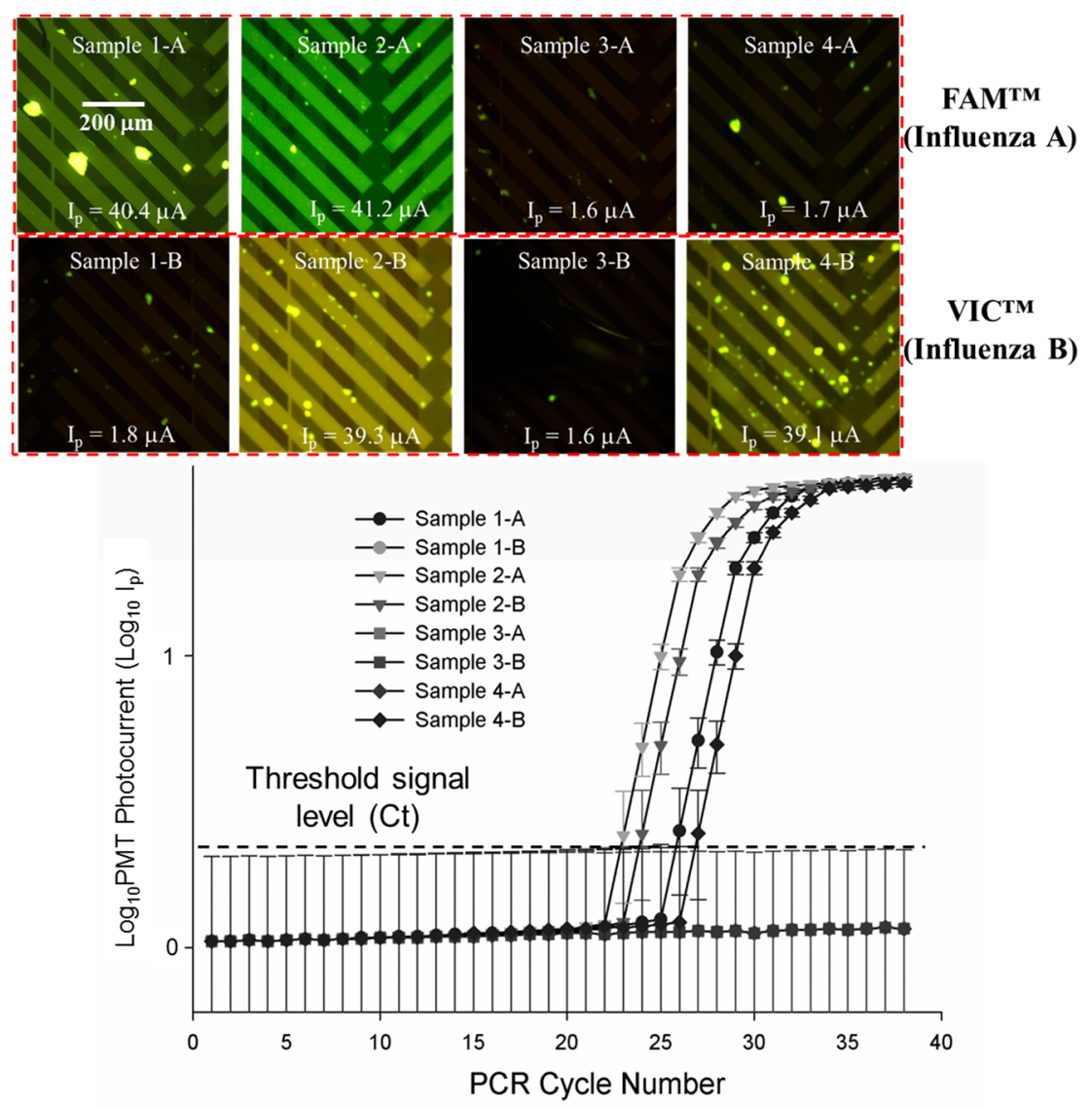

Figure 7. Photomicrographs showing the fluorescent images corresponding to the eight PCR droplets and the extracted plot of the eight qRT-PCR curves $\left(I_{\mathrm{p}}\right.$ in $\left.\mu \mathrm{A}\right)$.

Table 4. Outcomes of the micro-device qRT-PCR assay using mixed panel samples of Table 1c.

\begin{tabular}{ccccc}
\hline Panel Sample No. & Target & ProvLab $\boldsymbol{C} \boldsymbol{t}$ & Chip $\boldsymbol{C} \boldsymbol{t}$ & Initial Copies of Template RNA \\
\hline 1-A & Flu A & 29 & 27 & $\sim 290$ \\
1-B & Flu B & Negative & Negative & Not applicable \\
2-A & Flu A & 27 & 24 & $\sim 2900$ \\
2-B & Flu B & 28 & 25 & $\sim 1050$ \\
3-A & Flu A & Negative & Negative & Not applicable \\
3-B & Flu B & Negative & Negative & Not applicable \\
4-A & Flu A & Negative & Negative & Not applicable \\
4-B & Flu B & 30 & 28 & $\sim 110$ \\
\hline
\end{tabular}

\section{Conclusions}

This present investigation demonstrates and furthermore extends the applicability of the continuous D-DEP based droplet transport method for parallel, spatially multiplexed qRT-PCR reactions on a nano-textured DMF chip. The improved micro-electrode architecture accommodates up to eight parallel, qRT-PCR reactions. As a proof of principle, detection of Influenza A and B viruses from clinical samples was conducted using a blind panel. Influenza A and B were accurately identified and 
quantified using the standard quantification method, in the two micro-device based qRT-PCR assays. The outcomes of the repeated blind panel experiments confirm that the micro-device can successfully handle more than one nucleic acid samples and markers over an array of parallel, spatially multiplexed DMF micro-electrodes, to screen for a panel of viral/infectious diseases. The efficiency of chip based qRT-PCR assays were reasonably within the accepted industrial benchmark (PCR efficiency 94\%-97\%) and the completion time for the sample loading/mixing, RT-reactions and up to 38 PCR thermal cycles for up to eight different PCR droplets was found to be $\sim 35-40 \mathrm{~min}$, again comparable to that of a commercial fast qRT-PCR equipment. The detection limit, as identified using the chip based standard quantification process, for the multiplexed qRT-PCR micro-device was found to be $<10$ copies of RNA templates/PCR reaction. The micro-device furthermore offers future integration of both spatial (parallel qPCR reactions with differed targets) and spectral (multiple target markers in same PCR assay) multiplexing to screen for a larger panel of infectious agents. As a next step in the development, our focus is to improve the up-stream sample handling to achieve serial dilution of RNA samples and facilitate on-chip mixing and preparation of the reagent mixture and dispensing of multitude of sample droplets to suitably address the multiplexed qRT-PCR tracks. In addition, we will focus on the development of a separate sample extraction and purification chip to separate, lyse and concentrate target DNA/RNA from clinical patient samples, in preparation for the qRT-PCR amplification and detection stage. These proposed developments will lead to a portable sample-to-detection microsystem, suitable for example for field analysis of human, live-stock and food borne pathogens.

\section{Acknowledgments}

The authors gratefully acknowledge the financial support received from National Science and Engineering Research Council of Canada (NSERC) under the Discovery Grant program and that provided by CMC Microsystems (Canada) to support the microfabrication of the devices utilized in the study. The authors are furthermore thankful to the Provincial Laboratory for Public Health of Alberta for providing the extracted clinical samples and PCR reagents used in this work.

\section{Author Contributions}

Ravi Prakash designed and fabricated the DMF micro-chips, conducted the on-chip, qRT-PCR experiments, data analysis and contributed to the manuscript writing and revision process; Kanti Pabbaraju and Sallene Wong contributed in sample extraction, off-chip validation experiments and manuscript writing; Anita Wong prepared the extracted clinical samples and PCR reagents for both off-chip and on-chip qRT-PCR experiments and validated the micro-chip experimental data; Raymond Tellier contributed to the planning of the validation experiments and assisted in paper writing and revision; Karan Kaler contributions include the design of the developed DMF micro-chips; facilitated the DMF micro-chip based qRT-PCR experiments and contributed to the writing, revision and proofreading of the manuscript.

\section{Conflicts of Interest}

The authors declare no conflict of interest. 


\section{References}

1. Saiki, D.H.; Gelfand, S.; Stoffel, S.; Scharf, S.J.; Higuchi, R.; Horn, G.T.; Mullis, K.B.; Erlich, H.A. Primer-directed enzymatic amplification of DNA with a thermostable DNA polymerase. Science 1988, 239, 487-491.

2. Langin, T.; Robert, T. Mullis, K. B., Ferré, F. and Gibbs, R. A. (Eds.). 1994. The polymerase chain reaction (PCR). Birkhäuser Verlag AG, Basel, Switzerland. ISBN 3-7643-3607-2 (H.b.). ISBN 3-7643-3750-8 (P.b.). J. Evol. Biol. 1994, 8, 399-401.

3. Markoulatos, P.; Siafakas, N.; Moncany, M. Multiplex polymerase chain reaction: A practical approach. J. Clin. Lab. Anal. 2002, 16, 47-51.

4. Logan, J.; Edwards, K.J.; Saunders, N. Real-Time PCR: Current Technology and Applications; Caister Academic Press: Norfolk, UK, 2009.

5. Wittmer, C.T.; Kusukawa, N. Real-time PCR and melting analysis. In Molecular Microbiology Diagnostic Principles and Practice, 2nd ed.; ASM Press: Washington, DC, USA, 2011.

6. Kopp, M.U.; de Mello, A.J.; Manz, A. Chemical amplification: Continuous-flow PCR on a chip. Science 1998, 280, 1046-1048.

7. Manz, A.; Becker, H. Microsystem Technology in Chemistry and Life Sciences; Springer: Berlin, Germany, 1998.

8. Lagally, E.T.; Emrich, C.A.; Mathies, R.A. Fully integrated PCR-capillary electrophoresis microsystem for DNA analysis. Lab Chip 2001, 1-2, 102-107.

9. Liu, R.H.; Yang, J.N.; Lenigk, R.; Bonanno, J.; Grodzinski, P. Self-contained, fully integrated biochip for sample preparation, polymerase chain reaction amplification, and DNA microarray detection. J. Anal. Chem. 2004, 76, 1824-1831.

10. Tewhey, R.; Warner, J.B.; Nakano, M.; Libby, B.; Medkova, M.; David, P.H.; Kotsopoulos, S.K.; Samuels, M.L.; Hutchison, J.B.; Larson, J.W.; Topol, E.J.; Weiner, M.P.; Harismendy, O.; Olson, J.; Link, D.R.; Frazer, K.A. Microdroplet-based PCR enrichment for large-scale targeted sequencing. Nat. Biotechnol. 2009, 27, 1025-1031.

11. Prakash, R.; Pabbaraju, K.; Wong, S.; Wong, A.; Tellier, R.; Kaler, K.V.I.S. Droplet microfluidic chip based nucleic acid amplification and real-time detection of influenza viruses. J. Electrochem. Soc. 2014, 161, 3083-3093.

12. Dahl, A.; Sultan, M.; Jung, A.; Schwartz, R.; Lange, M.; Steinwand, M.; Livak, K.; Lehrach, H.; Nyarsik, L. Quantitative PCR based expression analysis on a nanoliter scale using polymer nano-well chips. Biomed. Microdev. 2007, 9, 307-314.

13. Freire, V.S.; Ebert, A.D.; Kalisky, T.; Quake, S.R.; Wu, J.C. Microfluidic single-cell real-time PCR for comparative analysis of gene expression patterns. Nat. Protoc. 2012, 7, 829-838.

14. Saunders, D.C.; Holst, G.L.; Phaneuf, C.R.; Pak, N.; Marchese, M.; Sondej, N.; McKinnon, M.; Forest, C.R. Rapid, quantitative, reverse transcription PCR in a polymer microfluidic chip. Biosens. Bioelec. 2013, 44, 222-228.

15. Lee, S.H.; Kim, S.W.; Kang, J.Y.; Ahn, C.H. A polymer lab-on-a-chip for reverse transcription (RT)-PCR based point-of-care clinical diagnostics. Lab Chip 2008, 8, 2121-2127. 
16. Roberts, C.H.; Jiang, W.; Jayaraman, J.; Trowsdale, J.; Holland, M.J.; Traherne, J.A. Killer-cell Immunoglobulin-like receptor gene linkage and copy number variation analysis by droplet digital PCR. Genome Med. 2014, 6, 1-9.

17. Pollack, M.G.; Fair, R.B.; Shenderov, A.D. Electrowetting-based actuation of liquid droplets for microfluidic applications. Appl. Phys. Lett. 2000, 77, 1725-1726.

18. Cho, S.K.; Moon, H.; Kim, C.J. Creating, Transporting, Cutting, and Merging Liquid Droplets by Electrowetting-Based Actuation for Digital Microfluidic Circuits. J. Microelectromech. Syst. 2003, $12,70-80$.

19. Sista, R.; Hua, Z.; Thwar, P.; Sudarsan, A.; Srinivasan, V.; Eckhardt, A.E.; Pollock, M.; Pamula, V. Development of a digital microfluidic platform for point of care testing. Lab Chip 2008, 8, 2091-2104.

20. Hua, Z.; Rouse, J.L.; Eckhardt, A.E.; Srinivasan, V.; Pamula, V.K.; Schell, W.A.; Benton, J.L.; Mitchell, T.G.; Pollack, M.G. Multiplexed Real-Time Polymerase Chain Reaction on a Digital Microfluidic Platform. Anal. Chem. 2010, 82, 2310-2316.

21. Srinivasan, V.; Pamula, V.K.; Fair, R.B. An integrated digital microfluidic lab-on-a-chip for clinical diagnostics on human physiological fluids. Lab Chip 2004, 4, 310-315.

22. Chang, Y.H.; Lee, G.B.; Huang, F.C.; Chen, Y.Y.; Lin, J.L. Integrated polymerase chain reaction chips utilizing digital microfluidics. Biomed. Microdev. 2006, 8, 215-225.

23. Gunji, M.; Nakanishi, H.; Washizu, M. Droplet actuation based on single-phase electrostatic excitation. In Proceedings of 8th International Conf. on Miniaturized Systems in Chemistry and Life Sciences ( $\mu$-TAS2004), Malmö, Sweden, September 26-30 2004; Volume 1, pp. 168-170.

24. Kaler, K.V.I.S.; Prakash, R.; Chugh, D. Liquid Dieletrophoresis and Surface Microfluidics. Biomicrofluidics 2010, 4, 022805.

25. Prakash, R.; Papageorgiou, D.P.; Papathanasiou, A.G.; Kaler, K.V.I.S. Dielectrophoretic liquid actuation on nano-textured super hydrophobic surfaces. Sens. Actuators B Chem. 2013, 182, 351-361.

26. Lagally, E.T.; Simpson, P.C.; Mathies, R.A. Monolithic integrated microfluidic DNA amplification and capillary electrophoresis analysis system. Sens. Actuators B Chem. 2006, 63, 138-146.

27. Zhong, R.; Pan, X.; Jiang, L.; Dai, Z.; Qin, J.; Lin, B. Simply and reliably integrating micro heaters/sensors in a monolithic PCR-CE microfluidic genetic analysis system. Electrophoresis 2009, 30, 1297-1305.

28. Pfaffl, M.W. A new mathematical model for relative quantification in real-time RT-PCR. Nucleic Acids Res. 2001, 29, 2002-2007.

(C) 2014 by the authors; licensee MDPI, Basel, Switzerland. This article is an open access article distributed under the terms and conditions of the Creative Commons Attribution license (http://creativecommons.org/licenses/by/4.0/). 